\title{
Novel reference materials for laser- induced breakdown spectroscopy [LIBS] microanalysis
}

\author{
SOMERS A.M. ${ }^{1 *}$., NORDSTAD $\mathrm{S}^{2}$. \\ ${ }^{1}$ SciAps Inc., 7 Constitution Way, Woburn, MA01801, USA \\ (asomers@sciaps.com) \\ 2 myStandards GmbH, Schauenburgerstr. 116, 24118 Kiel, \\ Germany (nordstad@my-standards.com)
}

Laser-induced breakdown spectroscopy (LIBS) is an emerging technique for rapid, in-situ, microanalysis that is ideally suited to the anlaysis of geoloigcal samples. The recent advent of portable LIBS instruments offers the possibility of use both in the field as well as in the laboratory. For quantification of acquired geochmeical data an empirical matrix-matched calibration is preferable (Harmon et al. [1]). However, reference materials which are sufficiently homogenous on the $\mu \mathrm{m}$-scale are scarce. The main issue with most commercially available reference materials is the coarse particle size ranging from $20-150 \mu \mathrm{m}$, which is close to the typical beam diameter of 50-100 $\mu \mathrm{m}$. The nature of sampling individual clasts within an inhomegeous material does not allow stoichiometric sampling and a large number of calibration shots for averaging are required. Using new patented techniques to reduce the particle size of most rocks and minerals to a $\mathrm{D}_{50}$ of $\sim 200 \mathrm{~nm}$ it is now possible to produce nano-particulate pressed powder pellets without any binders, which are homogenous at the $\mu \mathrm{m}$-scale and also exhibit superior ablation behaviour compared to its coarser counterparts. This study investigates the advantages of using these nano-particulate samples related to improved signal quality and efficiency in the number of measurements required to generate representative sampling. Also of interest is the impact on data quality when developing qauntitative calibrations and testing samples using nano-particulate materials as calibration standards as compared to conventionally prepared reference materials.

[1] Harmon et al. (2019) Minerals 9, 718. 